\title{
Preface to the special issue on concept lattices and their applications-CLA 2012
}

\author{
Uta Priss · Laszlo Szathmary
}

Published online: 8 March 2014

(C) Springer International Publishing Switzerland 2014

The Ninth International Conference "Concept Lattices and Applications (CLA 2012)" was held in Fuengirola (Málaga), Spain from October 11th until October 14th 2012. CLA is a conference series which was started in 2002 and which focuses on Concept Lattices, Formal Concept Analysis (FCA) and Galois Lattices. FCA was invented by Rudolf Wille in the 1980 s as a mathematical modeling of hierarchies, data analysis and conceptual descriptions using mathematical lattice theory. A binary relation (for example between some objects and their attributes) is modeled as a formal context which is transformed into a concept lattice. Because binary relations can be extracted from any relational database or other data repository, FCA has applications in many disciplines, such as data and knowledge processing, information retrieval, data mining, reasoning and software engineering. Since the 1980s, FCA has grown into a reasonably large international research community with two annual international conference series (CLA and ICFCA). Concept lattices can be graphically represented which encourages the development of graphical interfaces for end users and poses interesting research questions relating to graph theory. In the context of "big data", research questions focus on improving the efficiency of FCA algorithms and on decomposing formal contexts into meaningful smaller units. More recently there has been an increasing interest in Fuzzy FCA, which supports the application of FCA to non-discrete datasets and the factorization of binary matrices as a generalization of formal contexts.

This special issue contains the 9 best papers from CLA 2012. In total 44 papers were submitted to CLA 2012 out of which 28 papers were accepted as full papers and published as Volume 972 in the CEUR series (ceur-ws.org). For the special issue, the authors

U. Priss $(\bowtie)$

Zentrum für erfolgreiches Lehren und Lernen, Ostfalia University of Applied Sciences,

Wolfenbüttel, Germany

URL: www.upriss.org.uk

L. Szathmary

University of Debrecen, Debrecen, Hungary

e-mail: Szathmary.L@gmail.com 
of 13 papers were invited to submit extended versions of their papers of which 9 were ultimately accepted. The topics of the papers in this special issue can be grouped into three main areas. The first area is matrix factorization and reduction. Belohlavek, Outrata and Trnecka study how to improve the classification of Boolean data by finding relevant factorizations of the data. Belohlavek and Krmelova discuss matrix factorization of data with ordinal attributes. Berry, Gutierrez, Huchard, Napoli and Sigayret describe an algorithm for building a partial-order substructure of a concept lattice. The second topic area relates to fuzzy approaches. Konecny, Medina and Ojeda-Aciego suggest size reductions for fuzzy concept lattices. Kridlo and Ojeda-Aciego study fuzzy extensions of formal concept analysis in the context of category theory. Alcalde, Burusco and Fuentes-Gonzalez investigate the use of fuzzy FCA for morphological image and signal processing. The third topic area in this special issue is concerned with databases, data mining and retrieval. Baixeries, Kaytoue and Napoli consider the computation of functional dependencies in the context of FCA. Kwuida, Missaoui, Balamane and Vaillancourt extract patterns from data with taxonomic structures. Codocedo, Lykourentzou and Napoli suggest using FCA for semantic document indexing and retrieval.

We would like to thank the authors for their contributions, the members of the program and steering committees, the conference participants for their participation and support, the local organizers of the conference and, in particular, the reviewers both of the conference and this special issue without whose tremendous efforts neither the conference, nor the special issue would have been possible. We would also like to express gratitude for the financial support that this conference has received from the Research Promotion Programme of the Universidad de Málaga.

December 2013

Uta Priss

Laszlo Szathmary 